\title{
Potential applications of the dielectric wakefield accelerators in the SINBAD facility at DESY
}

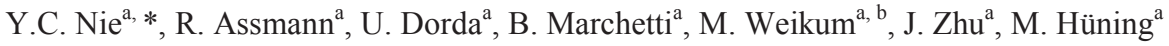 \\ ${ }^{a}$ Deutsches Elektronen-Synchrotron DESY, 22607 Hamburg, Germany \\ ${ }^{\mathrm{b}}$ University of Strathclyde, G1 1XQ Glasgow, United Kingdom
}

*Corresponding author at: DESY, Hamburg 22607, Germany. E-mail: yuancun.nie@desy.de

\begin{abstract}
Short, high-brightness relativistic electron bunches can drive ultra-high wakefields in the dielectric wakefield accelerators (DWFAs). This effect can be used to generate high power THz coherent Cherenkov radiation, accelerate a witness bunch with gradient two or three orders of magnitude larger than that in the conventional RF linear accelerators, and introduce energy modulation within the driving bunch itself, etc. The paper studies potential applications of the DWFAs in the SINBAD facility at DESY. The simulations show that the ultra-short relativistic bunches from the SINBAD injector ARES can excite accelerating wakefields with peak amplitudes as high as $\mathrm{GV} / \mathrm{m}$ at $\mathrm{THz}$ frequencies in proper DWFA structures. In addition, it illustrates that the DWFA structure can serve as a dechirper to compensate the correlated energy spread of the bunches accelerated by the laser plasma wakefield accelerator.
\end{abstract}

Keywords: Dielectric wakefield accelerator; Ultra-short electron bunch; Accelerating gradient; Energy spread.

\section{Introduction}

The dielectric accelerator is one of the most advanced accelerator concepts, in which the ultra-high accelerating field can be excited by either optical to infrared lasers or ultra-short relativistic electron bunches. Dielectric accelerators have promising applications in the future high energy colliders and free electron lasers, due to their compact sizes. For the dielectric laser accelerators [1-4], a number of structures have been studied, such as the grating structure, the 2D and 3D photonic band-gap structure, and so on. The beam driven dielectric wakefield accelerators (DWFAs) make use of the electromagnetic Cherenkov radiation (wakefield) from the electron bunches that pass through the dielectric lined waveguides (DLWs) [5]. The most used DWFA structure is the single layer cylindrical DLW. Besides, many other DLW structures have been investigated, for instances, the double-layer cylindrical DLW [6] and the rectangular (slab-symmetric) DLW [7]. DWFAs have wide applications in modern sciences. Narrow-band high-power THz radiation can be generated from the driving electron bunch directly in the DLWs $[8,9]$. The wakefield can be used to accelerate a witness bunch with gradient two or three orders of magnitude larger than that in conventional metal RF linear accelerators. The mechanism has been demonstrated experimentally at the AWA facility of ANL [10], the ATF facility of BNL [11] and the FACET facility of SLAC [12]. Moreover, the energy modulation of the driving bunch itself can be used to compensate its time-dependent energy spread like a dechirper or produce bunch trains indirectly [13]. For instance, based on the self-wake, a tunable energy dechirper was used to remove the correlated energy spread from the $60 \mathrm{MeV}$ beam at the ATF facility [14]. Such a dechirper may simplify linac beamline design significantly and improve the performance of the free electron lasers $[14,15]$.

The accelerator research and development (ARD) group at DESY is investigating a novel facility, the Short and INnovative Bunches and Accelerators at DESY (SINBAD) [16, 17]. Within the SINBAD facility, we will study the production of ultra-short relativistic electron bunches, the high gradient advanced accelerator concepts, and their various applications. Among a number of topics, one idea is the external injection experiment for the laser plasma wakefield acceleration (LWFA) [18]. The external bunches to be accelerated in the LWFA will be provided by ARES: Accelerator Research Experiment at SINBAD. Based on the conventional S-band and Xband RF cavities, ARES aims to generate ultra-short electron bunches having FWHM length from subfemtosecond to a few fs with relatively low charge ( $0.5 \mathrm{pC}$ to a few $\mathrm{pC}$ ) and energy up to $200 \mathrm{MeV}$. It is of great interest to explore the potential applications of the DWFAs in the SINBAD facility. For example, it is of great interest to use the ultra-short bunches from ARES to drive ultra-high gradient accelerating wakefields in a DWFA. On the other hand, the DLW structure may serve as a dechirper to compensate the positive energy chirp 
of a LWFA-accelerated bunch, in which the head electrons have lower energy than the tail electrons as long as the acceleration is terminated before dephasing $[19,20]$.

\section{Wakefield Analysis and Simulations}

The wakefield stimulated by the driving bunch in a DLW consists of a series of discrete electromagnetic modes depending on the bunch and waveguide parameters. Figure 1 shows the two commonly used DLWs, i.e. the cylindrical and the rectangular dielectric waveguides. In this paper, the dielectric material is CVD (chemical vapor deposition) diamond as proposed in [11]. It has been demonstrated in [11] and its previous works that this kind of material has a very low loss tangent $\left(<10^{-4}\right)$ in the Ka-W frequency bands, a thermal conductivity higher than $2000 \mathrm{~W} / \mathrm{K} / \mathrm{m}$ at room temperature and a DC breakdown threshold on the level of $1 \mathrm{GV} / \mathrm{m}$. Note that diamond is used in our current simulations temporarily. Some other dielectric materials might be selected in the future practical design. For example, fused silica is a potential candidate as an alternative, of which an accelerating field breakdown threshold of $8 \mathrm{GV} / \mathrm{m}$ has been observed at THz frequencies in [21]. The relative permittivity of diamond $\varepsilon_{r}$ is around 5.7. For the cylindrical structure, the inner radius and outer radius of the dielectric layer are denoted by $a$ and $b$, respectively. The frequency $f$ and wavelength $\lambda$ of the fundamental mode $\left(\mathrm{TM}_{01}\right)$ can be estimated by the following equation [21]:

$$
f=\frac{c}{\lambda}=\frac{c}{4(b-a) \sqrt{\varepsilon_{r}-1}}
$$

or by:

$$
f=\frac{c}{\lambda}=\frac{c}{2 \pi} \cdot \sqrt{\frac{\varepsilon_{r}}{\left(\varepsilon_{r}-1\right)(b-a) a}}
$$

when the thickness of the dielectric layer is much smaller than the inner radius of the DLW tube, i.e. $(b-a)<<a$. Compared with Ref. [22], the corrugated structure is replaced by the dielectric layer, so we have decreased the frequency by a factor of $\sqrt{2}$ in Eq. (2) based on the results in [23]. Meanwhile, the maximum longitudinal decelerating wakefield within the driving bunch $E_{z \text {,dec }}$, and the peak surface electric field $E_{r, \text { surf }}$ can be calculated approximately by the expression [21]:

$$
e E_{z, \mathrm{dec}}=e E_{r, \text { surf }} \frac{\sqrt{\varepsilon_{r}-1}}{\varepsilon_{r}} \cong \frac{4 N_{b} r_{e} m_{e} c^{2}}{a\left[\sqrt{\frac{8 \pi}{\varepsilon_{r}-1}} \varepsilon_{r} \sigma_{z}+a\right]},
$$

where $r_{e}$ and $m_{e} c^{2}$ are the classical radius and rest energy of the electron, respectively, $e$ is the absolute charge of one electron, $N_{b}$ is the number of electrons per bunch, and $\sigma_{z}$ is the rms bunch length. Substituting $r_{e}=$ $\frac{1}{4 \pi \varepsilon_{0}} \frac{e^{2}}{m_{e} c^{2}}$ and $N_{b}=Q / e$ into Eq. (3), we have:

$$
E_{z, \mathrm{dec}} \cong \frac{Q}{\pi \varepsilon_{0} a\left(\sqrt{\frac{8 \pi}{\varepsilon_{r}-1}} \varepsilon_{r} \sigma_{\mathrm{Z}}+a\right)},
$$

where $\varepsilon_{0}$ is the permittivity of free space and $Q$ is the total bunch charge.

Using Eqs. (1)-(4), a preliminary design of a DLW structure may be carried out. Meanwhile, there are a few numerical codes, such as OOPIC PRO [24], and RECTANGULAR for the rectangular DLWs [7]. In this paper, the Wakefield Solver of CST [25] is used. It assumes that the driving bunch has a line Gaussian distribution. The following bunch parameters are used: average energy $E=100 \mathrm{MeV}$, total bunch charge $Q=1 \mathrm{pC}$, bunch length $\sigma_{z}=0.3 \mu \mathrm{m}, 1.0 \mu \mathrm{m}$, or $3.0 \mu \mathrm{m}$. The longitudinal wake impedances and on axis wakefields are shown in Figs. 2 and 3 for two bunch-length cases using the cylindrical waveguide. It can be seen that the wake strength mainly depends on the inner radius of the dielectric layer for a certain driving bunch. The inner radius $a$ has to be small enough corresponding to the bunch length to excite significant wakefields, the outer radius $b$ has to be chosen properly as well to control the wakefield frequencies and hence the integrated waveforms. For instance, in the case of $\sigma_{z}=0.3 \mu \mathrm{m}$ as shown in Fig. 2, there is only weak wakefield excited when $a$ is $5 \mu \mathrm{m}$, but the accelerating wakefield becomes as high as $4.8 \mathrm{GV} / \mathrm{m}$ when $a$ is $2 \mu \mathrm{m}$. The wakefield frequencies are higher than 
$5 \mathrm{THz}$ in all the cases. In the case of $\sigma_{z}=3 \mu \mathrm{m}$ as shown in Fig. 3, the wake is relatively weak. However, when the inner radius $a$ is $20 \mu \mathrm{m}$, the decelerating wakefield magnitude within the driving bunch increases linearly along the bunch from head to tail. Such a time-dependent wake is very suitable for compensating the positive energy chirp, since the trailing electrons that have higher energy will lose more energy than the head ones. In the next section, the so called energy spread dechirper will be described in detail. Table 1 lists the related theoretical results and simulation values. Good agreements can be observed. As illustrated in the table, the transformer ratio defined as the ratio of the maximum energy gain of the witness bunch to the maximum energy loss of the driving bunch, i.e. $R=E_{z, \text { acc }} / E_{z, \text { dec }}$, is generally lower than 2 unless certain specific techniques are used [26].
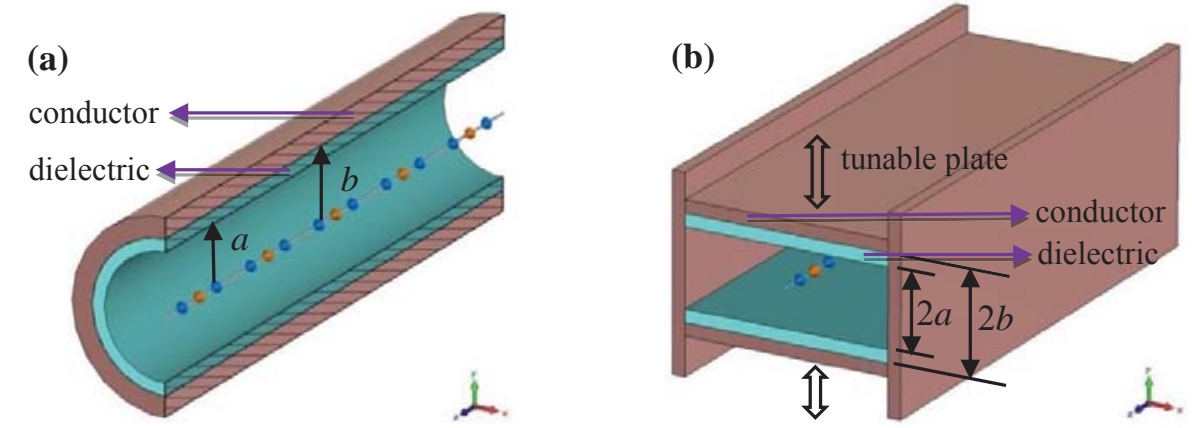

Fig. 1. (a) Cylindrical dielectric waveguide and (b) Rectangular dielectric waveguide.
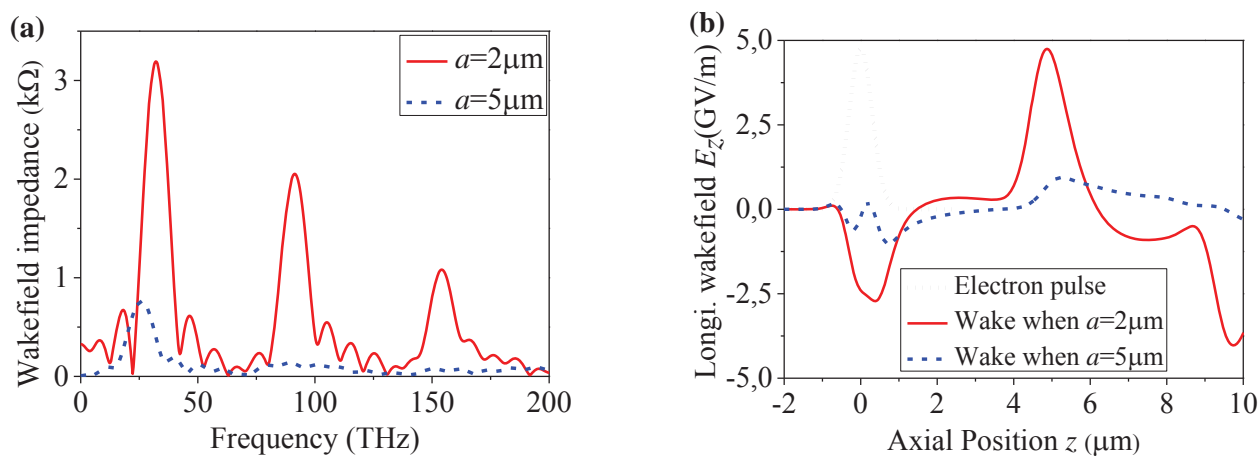

Fig. 2. Longitudinal wake impedance vs. frequencies (a), and wakefield vs. distance after the driving bunch (b). $\sigma_{\mathrm{z}}$ is $0.3 \mu \mathrm{m}, Q$ is $1 \mathrm{pC}$. The inner radius $a$ is 2 or $5 \mu \mathrm{m}$, while the dielectric layer thickness is kept to be $1 \mu \mathrm{m}$.
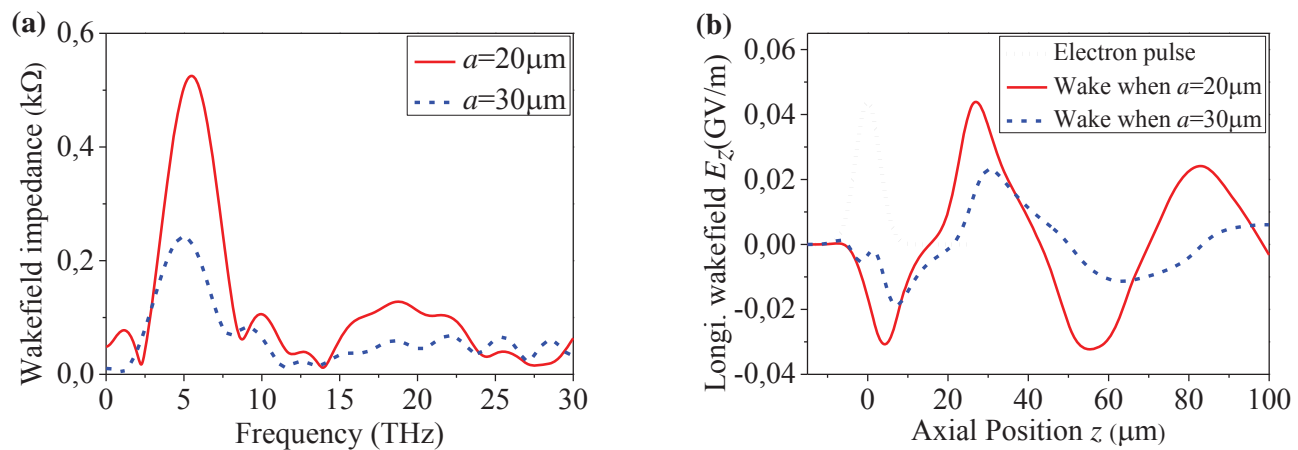

Fig. 3. Longitudinal wake impedance vs. frequencies (a), and wakefield vs. distance after the driving bunch (b). $\sigma_{\mathrm{z}}$ is $3.0 \mu \mathrm{m}, Q$ is $1 \mathrm{pC}$. The inner radius $a$ is 20 or $30 \mu \mathrm{m}$, while the dielectric layer thickness is kept to be $5 \mu \mathrm{m}$.

Table 1 Comparisons between the theoretical results and the simulated ones.

\begin{tabular}{llllllllll}
\hline $\begin{array}{l}\sigma_{\mathrm{z}} \\
(\mu \mathrm{m})\end{array}$ & $\begin{array}{l}\boldsymbol{Q} \\
(\mathrm{pC})\end{array}$ & $\begin{array}{l}a \\
(\mu \mathrm{m})\end{array}$ & $\begin{array}{l}b \\
(\mu \mathrm{m})\end{array}$ & $\begin{array}{l}f_{\mathrm{TM} 01, \mathrm{Eq} .1} \\
(\mathrm{THz})\end{array}$ & $\begin{array}{l}f_{\mathrm{TM} 01, \mathrm{Eq} .2} \\
(\mathrm{THz})\end{array}$ & $\begin{array}{l}f_{\mathrm{TM} 01, \mathrm{CST}} \\
(\mathrm{THz})\end{array}$ & $\begin{array}{l}E_{z, \mathrm{dec} \text { Eq.4 }} \\
(\mathrm{GV} / \mathrm{m})\end{array}$ & $\begin{array}{l}E_{z, \text { dec, CST }} \\
(\mathrm{GV} / \mathrm{m})\end{array}$ & $\begin{array}{l}E_{z, \text { acc, CST }} \\
(\mathrm{GV} / \mathrm{m})\end{array}$ \\
\hline 0.3 & 1 & 2 & 3 & 34.6 & 37.2 & 32.5 & 3.019 & 2.730 & 4.750 \\
1 & 1 & 5 & 6 & 34.6 & 23.5 & 25.6 & 0.395 & 0.354 & 0.718 \\
3 & 1 & 20 & 25 & 6.9 & 5.2 & 5.5 & 0.030 & 0.031 & 0.044 \\
\hline
\end{tabular}




\section{Dechirper Studies}

In the LWFA, the witness bunch must be injected into a certain $90^{\circ}$ phase range of the plasma wave to accelerate and focus it simultaneously. As a result, the head electrons always gain less energy than the tail ones before the dephasing happens [20]. One solution is to remove the correlated energy spread using the self-wake of the bunch when it travels through a metallic corrugated pipe [27-30] or a DLW structure [13, 14].

For a dechirper, the rectangular dielectric structure is employed taking advantage of its continuously tunable gap as shown in Fig. 1 (b). We assume the output bunch parameters after the LWFA as follows: the average energy $E=200 \mathrm{MeV}$, the positive correlated rms energy spread Cor $E=2 \mathrm{MeV}$, the slice energy spread is \pm 0.3 $\mathrm{MeV}$, the Gaussian bunch size $\sigma_{x, y, z}=3.0 \mu \mathrm{m}$, and the bunch charge $Q$ is variable from $1 \mathrm{pC}$ to as high as $100 \mathrm{pC}$. Fig. 4 plots the impedances of the wakefields and their on-axis amplitudes when half the height of the vacuum chamber $a$ is $10 \mu \mathrm{m}, 15 \mu \mathrm{m}$ or $20 \mu \mathrm{m}$ and the dielectric layer thickness is kept to be $5 \mu \mathrm{m}$ when $Q=1 \mathrm{pC}$. In the case of $a=20 \mu \mathrm{m}$, the wake strength becomes $70 \%$ of the value in Fig. 3 due to different geometrical boundaries, differing with the prediction of $\pi^{2} / 16 \approx 60 \%$ in [30] for the corrugated structures, whereas the frequency drops by $10 \%$, not as much as $1-1 / \sqrt{2} \approx 30 \%$ as in [30]. Simulations with the Particle-In-Cell (PIC) Solver of CST have been performed as well. The size $a$ was selected to be $15 \mu \mathrm{m}$ to let the bunch pass through. Figure 5 (a) shows the energy distributions before and after a $750 \mu \mathrm{m}$ dechirper when $Q=100 \mathrm{pC}$. It can be seen that the correlated energy spread is compensated dramatically. The increment in the uncorrelated energy spread (slice energy spread) is observed. The reason is considered to be that the off axis electrons undergo various longitudinal and transverse wakefields strengths. There exists an optimal dechirper length to make the energy spread minimum, as shown in Fig. 5 (b), where we can see that the optimal dechirper length is around $750 \mu \mathrm{m}$. The peak magnitude in the optimized energy spectrum (peak $c$ ) is 2.5 times the initial one (peak $a$ ). The average energy is reduced by $2.5 \mathrm{MeV}$ in $750 \mu \mathrm{m}$, which implies a wake $E_{z, \mathrm{dec}}=3333 \mathrm{MV} / \mathrm{m}$ and a dechirper strength $S_{\mathrm{d}}$ $=1.9 \mathrm{MV} / \mathrm{m} / \mu \mathrm{m} / \mathrm{pC}$, where $S_{\mathrm{d}}$ is the normalized wakefield strength regarding the full bunch length and charge as defined in [14]. The wakefield strength in the PIC solver is in accordance with that in the Wakefield solver. It should be mentioned that the $100 \mathrm{pC}$ bunch charge is hardly to obtain for such short electron bunches. A higher charge allows us to compensate Cor_E sufficiently within a shorter dechirper length and hence get rid of the limitation of the computer memory, supposing that the wake strength is proportional to the bunch charge and the space charge effect can be ignored within a few $\mathrm{mm}$ transport distance. As a crosscheck, simulations were carried out for $10 \mathrm{pC}$ using a moderately long dechirper. The average energy was reduced by $1 \mathrm{MeV}$ after 3000 $\mu \mathrm{m}$, leading to a wake $E_{z, \mathrm{dec}}=333.3 \mathrm{MV} / \mathrm{m}$ and a dechirper strength $S_{\mathrm{d}}=1.9 \mathrm{MV} / \mathrm{m} / \mu \mathrm{m} / \mathrm{pC}$. The dechirper strength was the same as the value in the case of $100 \mathrm{pC}$. It implied that the optimal dechirper length was around $7500 \mu \mathrm{m}$ for $10 \mathrm{pC}$.
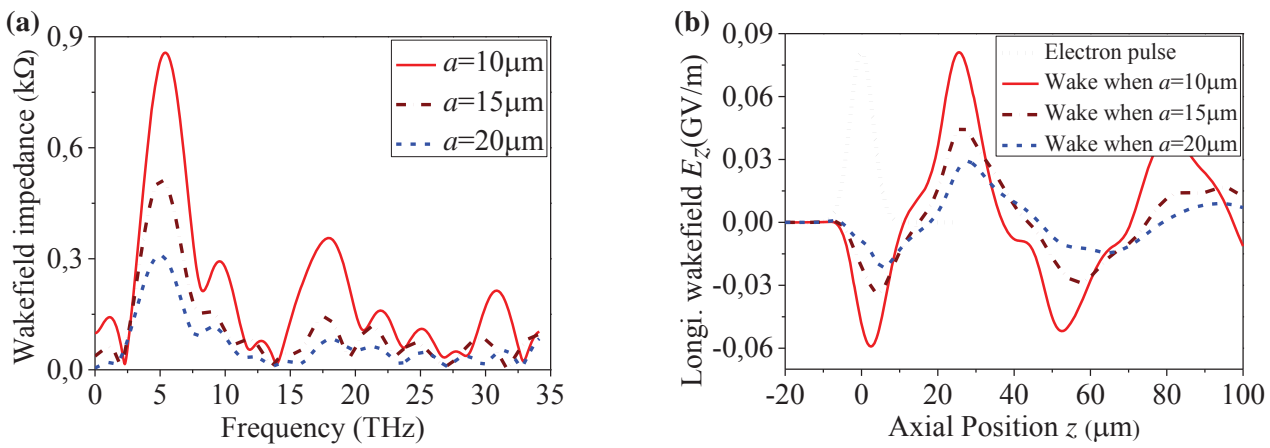

Fig. 4. Longitudinal wake impedance vs. frequencies (a), and wakefield vs. distance after the driving bunch (b). $\sigma_{\mathrm{z}}$ is $3.0 \mu \mathrm{m}, Q$ is $1 \mathrm{pC}$, as like in the case of Fig. 3. The rectangular waveguide structure is used here. Half height $a$ is 10,15 or $20 \mu \mathrm{m}$, while the dielectric layer thickness is kept to be $5 \mu \mathrm{m}$. 

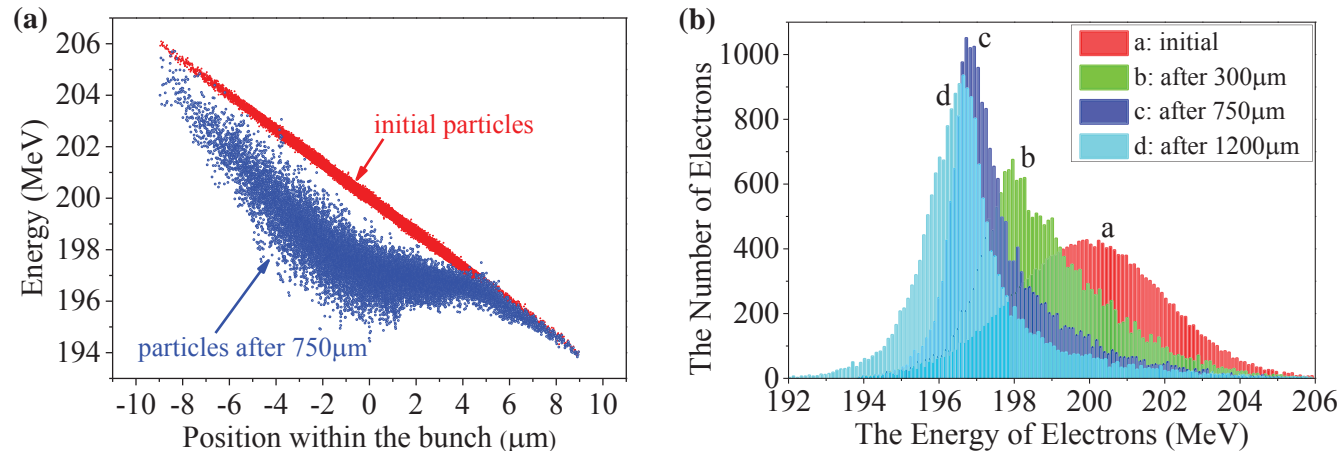

Fig. 5. Energy distributions before and after travelling $750 \mu \mathrm{m}$ in the dechirper (a), and energy spectrums at different dechirper lengths (b). A bunch charge $Q=100 \mathrm{pC}$ is used.

\section{Summary and outlook}

The paper has presented multiple applications of the DWFA structures in the SINBAD facility. The ultra-high accelerating wakefields up to $\mathrm{GV} / \mathrm{m}$ at tens of $\mathrm{THz}$ can be generated by the ultra-short electron bunches from ARES. Proof-of-concept simulations have shown that the DLW-based dechirper has great potential to compensate the correlated energy spread of the LWFA-accelerated bunch. In the simulation results, we noticed a transverse emittance growth by a few percent due to the time-dependent quadrupole wake along the bunch (it was found that the bunch tail was focused in $x$ direction, but not visibly in $y$ direction). Further efforts need to be made to minimize the increment in the slice energy spread and the emittance growth by optimizing the DLW structure and manipulating the transverse bunch size. The realization of such tiny structures is quite challenging. In the future, a more realistic initial bunch distribution from the LWFA will be employed to evaluate the overall performance of a DLW-based dechirper and to consider the practical difficulties.

\section{Acknowledgments}

Many thanks to Dr. C. Jing from Euclid Techlabs, USA, and Dr. G. Xia from University of Manchester and Cockcroft Institute, UK, for the helpful discussions.

\section{References}

[1] R. Joel England, et al., Dielectric laser accelerators, Review of Modern Physics 86 (2014) 1337-1389.

[2] Peter Bermel, Robert L. Byer, Eric R. Colby, et al., Summary of the 2011 dielectric laser accelerator workshop, Nucl. Instrum. Methods Phys. Res. A 734 (2014) 51-59.

[3] E. A. Peralta, K. Soong, R. J. England, et al., Demonstration of electron acceleration in a laser-driven dielectric microstructure, Nature 503 (2013) 91-94.

[4] John Breuer and Peter Hommelhoff, Laser-based acceleration of nonrelativistic electrons at a dielectric structure, Phys. Rev. Lett. 111 (2013) 134803.

[5] W. Gai, P. Schoessow, B. Cole, et al., Experimental demonstration of wake-field effects in dielectric structures, Phys. Rev. Lett. 61 (1988) 2756.

[6] A. Kanareykin, Dielectric Based Accelerator: Subpicosecond Bunch Train Production and Tunable Energy Chirp Correction, in: 1st European Advanced Accelerator Concepts Workshop, La Biodola, Isola d'Elba, Italy, 2013.

[7] S.S. Baturin, I.L. Sheinman, A.M. Altmark, and A.D. Kanareykin, Transverse operator method for wakefields in a rectangular dielectric loaded accelerating structure, Phys. Rev. ST Accel. Beams 16 (2013) 051302 .

[8] A.M. Cook, R. Tikhoplav, S.Y. Tochitsky, et al., Observation of narrow-band terahertz coherent Cherenkov radiation from a cylindrical dielectric-lined waveguide, Phys. Rev. Lett. 103 (2009) 095003.

[9] G. Andonian, O. Williams, X. Wei, et al., Resonant excitation of coherent Cerenkov radiation in dielectric lined waveguides, Appl. Phys. Lett. 98 (2011) 202901.

[10] C. Jing, A. Kanareykin, J.G. Power, et al., Observation of enhanced transformer ratio in collinear wakefield acceleration, Phys. Rev. Lett. 98 (2007) 144801. 
[11] S. Antipov, C. Jing, A. Kanareykin, et al., Experimental demonstration of wakefield effects in a THz planar diamond accelerating structure, Appl. Phys. Lett. 100 (2012) 132910.

[12] B.O'Shea, O. Williams, G. Andonian, et al., Observation of $>\mathrm{GV} / \mathrm{m}$ decelerating fields in dielectric lined waveguides, in: Proceedings of LINAC2014, Geneva, Switzerland, 2014.

[13] S. Antipov, C. Jing, M. Fedurin, et al., Experimental observation of energy modulation in electron beams passing through terahertz dielectric wakefield structures, Phys. Rev. Lett. 108 (2012) 144801.

[14] S. Antipov, S. Baturin, C. Jing, et al., Experimental demonstration of energy-chirp compensation by a tunable dielectric-based structure, Phys. Rev. Lett. 112 (2014) 114801.

[15] Haixiao Deng, Meng Zhang, Chao Feng, et al., Experimental demonstration of longitudinal beam phasespace linearizer in a free-electron laser facility by corrugated structures, Phys. Rev. Lett. 113 (2014) 254802.

[16] R. Assmann, C. Behrens, R. Brinkmann, et al., SINBAD - a proposal for a dedicated accelerator research facility at DESY, in: Proceedings of IPAC2014, Dresden, Germany, 2014.

[17] M. Barbara, et al., Electron-beam manipulation techniques in the SINBAD linac for external injection in plasma wakefield acceleration, these Proceedings.

[18] J. Grebenyuk, R. Assmann, U. Dorda and B. Marchetti, Laser-driven acceleration with external injection at SINBAD, in: Proceedings of IPAC2014, Dresden, Germany, 2014.

[19] R. Assmann, A European perspective on plasma acceleration, in: Workshop on Plasma Acceleration, London, 2014.

[20] A. R. Maier, A. Meseck, S. Reiche, et al., Demonstration scheme for a laser-plasma-driven free-electron laser, Phys. Rev. X 2 (2012) 031019.

[21] M.C. Thompson, H. Badakov, A.M. Cook, et al., Breakdown limits on gigavolt-per-meter electron-beamdriven wakefields in dielectric structures, Phys. Rev. Lett. 100 (2008) 214801.

[22] M. Hüning, H. Schlarb, P. Schmüser and M. Timm, Measurements of harmonic wake fields excited by rough surfaces, Phys. Rev. Lett. 88 (2002) 074802.

[23] Y. Nie, Wakefields in THz cylindrical dielectric lined waveguides driven by femtosecond electron bunches, Radiation Physics and Chemistry 106 (2015) 140-144.

[24] D.L. Bruhwiler, R.E. Giacone, J.R. Cary, et al., Particle-in-cell simulations of plasma accelerators and electron-neutral collisions, Phys. Rev. ST Accel. Beams 4 (2001) 101302.

[25] CST Software, 〈https://www.cst.com $\rangle$.

[26] J.G. Power, W. Gai, P. Schoessow, Wakefield excitation in multimode structures by a train of electron bunches, Phys. Rev. E 60 (1999) 6061.

[27] P. Emma, M. Venturini, K.L.F. Bane, et al., Experimental demonstration of energy-chirp control in relativistic electron bunches using a corrugated pipe, Phys. Rev. Lett. 112 (2014) 034801.

[28] K.L.F. Bane and G. Stupakov, Corrugated pipe as a beam dechirper, Nucl. Instrum. Methods Phys. Res. A 690 (2012) 106-110.

[29] Feichao Fu, Rui Wang, Pengfei Zhu, et al., Demonstration of nonlinear-energy-spread compensation in relativistic electron bunches with corrugated structures, Phys. Rev. Lett. 114 (2015) 114801.

[30] Zhen Zhang, Karl Bane, Yuantao Ding, et al., Electron beam energy chirp control with a rectangular corrugated structure at the Linac Coherent Light Source, Phys. Rev. ST Accel. Beams 18 (2015) 010702. 